\title{
Strength and aesthetics for consistency of manufacture in every type of restoration
}

When it comes to dental prosthetics and restorations, laboratories, dentists and patients all want the same thing: a natural, aesthetic result. Traditionally, functional and aesthetic needs have been best met by metal-ceramic bonded crowns, and for patients suffering from bruxism or those with worn-down dentition, metallic or metal-supported restorations often represented the only method of guaranteeing a reliable restorative result.

However, alongside advances in digital technology, dental materials have also developed and there is now a strong, highly aesthetic biocompatible alternative to metalceramic restorations, in the form of a highly translucent zirconium oxide, which can be used by laboratories to give their clients high quality consistent restorations every time.

Under the brand name Zirlux 16+ this Zirconia is an excellent, fully biocompatible material, which with a flexural strength of 1,200 MPa offers the technician unparalleled flexural strength combined with exceptional translucency through a simple digital workflow.

Zirlux $16+$ is available in all 16 Vita shades along with three bleach shades: OM1, OM2 and OM3. This is also combined with seven modifiers available in pink, purple, pink-purple for tissue shading and orange-brown, grey and light grey for gingival and incisal enhancement. Alongside white Zirconia discs and the Zirlux colouring liquids for bespoke shading through dipping, Zirlux $16+$ offers the technician a full range of options.

As an example of the quality of this material a $1 \mathrm{~mm}$ thick section of Zirlux $16+$ achieves $44 \%$ translucency, providing excellent, lifelike aesthetics.

Its lithium content means that Zirlux 16+ pre-shaded discs offer a highly translucent material suitable for full contour crowns and bridges as well as frameworks up to 14 units. It can also be used for implant abutments with a titanium base insert. The pre-shaded discs are isostatically pressed, which ensures the discs give consistent results in terms of strength, shade and aesthetics both in manufacture and finished restorations.

Thanks to its smooth occlusal surface, Zirlux $16+$ causes minimal wear to opposing enamel when polished, presenting an ideal solution for patients regardless of the clinical situation. This variety of options means that patients can be offered an aesthetic all-ceramic alternative to metal restorations that re-creates the character and translucency of natural teeth.

The Zirlux system is an integral part of Henry Schein ConnectDental, a complete solution focusing on the digitisation of dentistry and the digital workflow between the practice and the laboratory. Henry Schein ConnectDental relies on open systems which offer individual solutions for dental practices and laboratories that simplify processes and improve efficiency.

Laboratory owner Stephen Green explained why he has converted clients from metal ceramic restorations to Zirlux 16+ and the benefits this offers to all stakeholders.

He said: 'All our work is now through a digital workflow with all-ceramic restorations, a high percentage of which is Zirconia, for which we use Zirlux 16+. Using this material we create full contour restorations for stain and glaze as well as with a digital cut-back for micro-layering. We use Zirlux 16+ for large cases in which we are producing screw retained implant work on Ti-bases through to simple crowns. The efficiency and accuracy provided by this material is excellent and my clients love the predictability of combining this material with CADCAM manufacture, producing results of outstanding consistency time after time.

To enable patients to benefit from the strength and aesthetics of Zirlux 16+, dentists should request Zirlux 16+ restorations from their laboratory.

\section{Comparison chart}

50

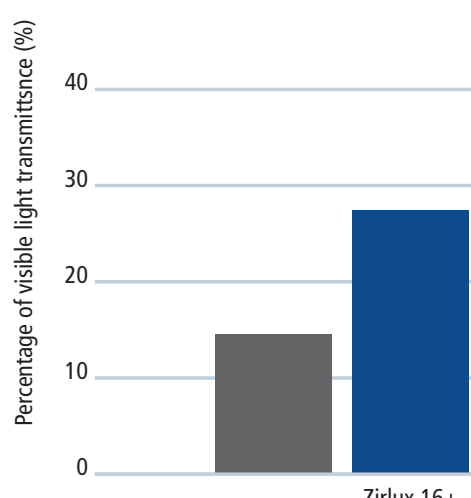

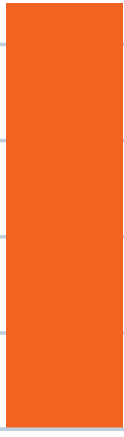

Zirlux $16+$

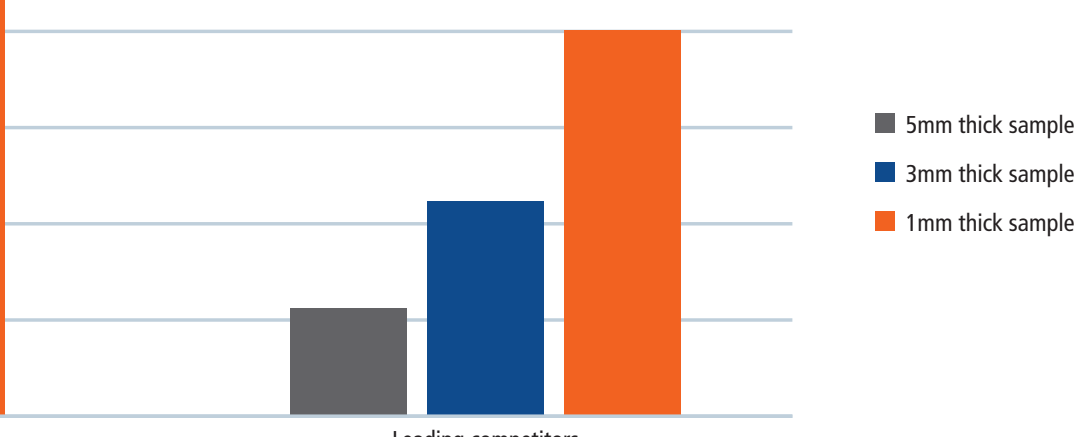

Leading competitors 